\title{
Erratum to: MEKC-LIF for Sensitive Discrimination of Six Potential Risk Predictors for Auxiliary Diagnosis of Anesthesia Complications in Clinical Urine Fluids
}

\author{
Fuwei $\mathrm{Qi}^{1} \cdot \mathrm{Hong} \mathrm{Xie}^{2} \cdot \mathrm{Zhong}^{\mathrm{Zheng}}{ }^{1} \cdot$ Yanhong Shang $^{1} \cdot \mathrm{Xiaohua} \mathrm{Fan}^{3} \cdot$
}

Xiuhua Zhao ${ }^{1}$

Published online: 21 January 2017

(C) Springer-Verlag Berlin Heidelberg 2017

\section{Erratum to: Chromatographia (2016)}

\section{9:1665-1670}

DOI 10.1007/s10337-016-3175-0

In the original publication, the address for the authors were incorrectly published.

The correct address list for the authors are:

${ }^{1}$ Department of Anesthesia, Taicang Affiliated Hospital of Soochow University, Taicang 215400, China

${ }^{2}$ Department of Anesthesia, The Second Affiliated Hospital of Soochow University, Suzhou 215004, China

${ }^{3}$ Department of Anesthesiology, Changhai Hospital, The Second Military Medical University, Shanghai 200433, China

The online version of the original article can be found under doi:10.1007/s10337-016-3175-0.

Xiaohua Fan

fxh95007@126.com

$\triangle$ Xiuhua Zhao

zjq53505070@126.com

1 Department of Anesthesia, Taicang Affiliated Hospital of Soochow University, Taicang 215400, China

2 Department of Anesthesia, The Second Affiliated Hospital of Soochow University, Suzhou 215004, China

3 Department of Anesthesiology, Changhai Hospital, The Second Military Medical University, Shanghai 200433, China 